\title{
Congenital Hypothyroidism in Child with Down syndrome - A Case Report
}

\author{
Halyna Bulak* and Yaryna Kozak
}

${ }^{1}$ Danylo Halytsky Lviv National Medical University, Ukraine

ISSN: 2576-9200

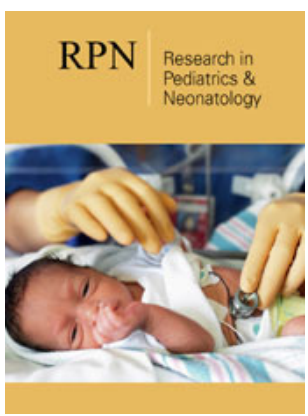

*Corresponding author: Halyna Bulak, Department of Pediatrics, Lviv City Children Hospital, Ukraine

Submission: 阱January 03, 2020

Published: 㘹January 28, 2020

Volume 4 - Issue 2

How to cite this article: Halyna B, Yaryna K. Congenital Hypothyroidism in Child with Down syndrome - A Case Report. Res Pediatr Neonatol. 4(2).RPN.000585.2020. DOI: $10.31031 / R P N .2020 .04 .000585$

\section{Copyright $(C)$ Halyna Bulak}

This article is distributed under the terms of the Creative Commons Attribution 4.0 International License, which permits unrestricted use and redistribution provided that the original author and source are credited.

\begin{abstract}
Congenital hypothyroidism is one of the most common congenital pathology of endocrine system in newborns. Among other diseases causing nervous system disorders in children, congenital hypothyroidism provides most promising treatment results for further child development. The earlier replacement therapy is started, the better results can be expected. This in its turn can only be possible with early diagnostics, mostly on subclinical stages, by screening newborns for this pathology. This article represents a clinical case of congenital hypothyroidism diagnosed on its early stage.
\end{abstract}

Keywords: Congenital hypothyroidism; Down syndrome; Hydrocephaly; Jaundice

Abbreviations: TSH-Thyroid Stimulating Hormone; T3-Triiodothyronine; T4-hyroxine; L-ThyroxineLevothyroxine, umol/l -Micromole Per Liter, nmol/l-Nanomole Per Liter

\section{Introduction}

Congenital hypothyroidism in newborns is characterized by lack of thyroid gland hormones, which play an important role in brain formation and development during pre- and postnatal period. Decrease in hormone levels can lead to mental retardation and permanent neurological disorders manifesting themselves in lack of coordination, ataxia, hypotonia. In most cases its clinical representation after birth is hardly distinctive and nonspecific: decrease in activity, lethargy, slow weight gain, constipation, prolonged jaundice. Early diagnostics is extremely important for the prevention of permanent brain damage by means of proper treatment. The implementation of screening programs for newborns in many countries has become a significant step forward for early diagnostics.

\section{Case Presentation}

Described below is a medical case of a child suffering from congenital hypothyroidism. At the age of six days the patient was hospitalized to newborn pathology department of Lviv City Children hospital having complaints of significant jaundice and discharge from the eyes. According to life history, on $18^{\text {th }}$ week of pregnancy ultrasound helped diagnose the fetus with hydrocephaly and Down syndrome was confirmed after amniocentesis had been performed. The mother's medical history did not contain any complaints during pregnancy. Child was delivered by caesarean section due to hydrocephaly. Newborn condition was moderate, Apgar score at birth reached $8 / 8$ points, body weight- 3280 grams, length $-50 \mathrm{~cm}$. Jaundice appeared on the second day of life; therefore, phototherapy was performed at the maternity hospital (bilirubin level before discharge-330umol/l). On the sixth day of life by neonatologist`s referral the patient was transferred to Lviv City Children hospital to be examined by neurosurgeon and further observed regarding jaundice.

During admission to Lviv City Children hospital the patient was in a stable state. Physical examination revealed a body temperature of $36,6^{\circ} \mathrm{C}$, heart rate of 148 beats/minute, respiratory rate of 48 breaths/minute, body weight- 3150 grams, physiological body position, hypotonia, suppressed reflexes, cold, mottled skin, acrocyanosis; jaundice; pallor of the mucous ; watery eye discharge, including light yellow mucus component. Stigmas of dysembriogenesisphenotype features of Down syndrome. Pulmonary auscultation was normal. Cardiovascular auscultation revealed sonorous, rhythmic heart tones, a systolic murmur radiating in the back. Liver palpating up to $2.0 \mathrm{~cm}$ below the costal arch. The urogenital system was developed in male type, testicles were in scrotum. Urinary output was spontaneous. Stool formed without 
pathological impurities. Laboratory analysis revealed normal blood and urine tests. Liver function tests showed total bilirubin level at $305 \mathrm{umol} / \mathrm{l}$. Bacteriological analysis of eye`s discharge was negative.

Neuro sonography demonstrated signs of congenital hydrocephaly (size of lateral ventricles: right - frontal horn $20 \mathrm{~mm}$, body of the ventricle $38 \mathrm{~mm}$, occipital horn $46 \mathrm{~mm}$, temporal horn $12 \mathrm{~mm}$; left - frontal horn $21 \mathrm{~mm}$, body of the ventricle $40 \mathrm{~mm}$, occipital horn $38 \mathrm{~mm}$, temporal horn $10 \mathrm{~mm}$; third ventricle $15 \mathrm{~mm}$ ), absence of cavum septum pellucidum and residual signs of subependymal hemorrhages (I grade). Echocardiography revealed a hemodynamically insignificant patent ductus arteriosus (2mm) and a patent foramen ovale $(4 \mathrm{~mm})$. Abdominal ultrasonography was normal. Neurological examination revealed diffuse muscular hypotony and hyporeflexia. Head circumference was $36 \mathrm{~cm}$, posterior fontanelle dimensions were $4,5 * 4 \mathrm{~cm}$. Monitoring by regular neuro sonography and symptomatic therapy was recommended. Measurement of thyroid gland hormone levels and thyroid-stimulating hormone (TSH) were performed so as to identify the cause of prolonged jaundice (Results -T3-2,6nmol/l, T4-164,3nmol/l, TSH - 22,38mm0/l, when normal values are T3 - 1,2-2,3nmol/l, T4 -11,5-23nmol/l, TSH - 0,177- 4,05mmo/l). Endocrinologist diagnosed congenital hypothyroidism. Treatment with Levo thyroxin was initiated in dosage of $12,5 \mathrm{mcg}$ daily for fourteen days with regular measurement of hormone levels to settle the issue of further replacement therapy. Based on all clinical data, physical examination as well as laboratory and instrumental examination, the final diagnosis for this patient was neonatal jaundice, congenital hydrocephaly, Down syndrome, conjunctivitis, congenital hypothyroidism, congenital heart defect (patent arterial duct and patent foramen ovale).

Moderate improvement of the child's condition was observed on day 10 of treatment, the yellowing of the skin and discharge from the eyes disappeared. Regular neuro sonography sessions showed slightly better dynamics with no signs of intraventricular hypertension. Measurement of thyroid hormone and TSH levels in blood were performed on day 14 of L-thyroxine therapy (Results: TSH-0,76mm0/l, T4-15,8nmol/l, T3-7,9nmol/l) showed evidence effectiveness and relevancy of prescribed treatment. At the age of twenty-six days, the child was discharged home in good condition. Parents received some important recommendations with regard to balanced nutrition and childcare. It was recommended to monitor head circumference once per ten days, to administer L-thyroxine in dosage of $12,5 \mathrm{mcg}$ once a day for ten days, to monitor hormone levels, to have a neurosurgical and genetical follow-up.

\section{Discussion}

Reduced synthesis of thyroid hormone in case of congenital hypothyroidism can be caused by disorders in thyroid gland development (dysgenesis) or of hormone biosynthesis processes. In addition, the lack of TSH can also serve as a reason, but it is more likely to be accompanied by deficiency of other pituitary gland hormones.Clinical features on early stages are often subtle. This can be explained by passage of maternal thyroid hormones across placenta, which provide protective effect to fetal brain as well as by moderate function of fetal own thyroid gland [1]. The most common signs include hypotonia, prolonged jaundice (due to immaturity of hepatic glucuronyl transferase), lethargy, feeding difficulties and constipation [2]. During examination such symptoms as cold mottled skin, macroglossia, umbilical hernia or wide posterior fontanel up to $5 \mathrm{~mm}$ draw the attention. During neurologic examination hypotonia and delayed reflexes can be noticed. Morbidity range widely varies by geographic location. That can be explained by endemic state of certain territories based on their iodine deficiency (e.g. in the USA the number is approximately 1 case per 2000-4000 newborns [3], whereas in Europe-according to ESPE (European Society for Pediatric Endocrinology) -1 case per 3000 newborns, in Ukraine-0,14 disease occurrence per 1000 newborns).

Congenital hypothyroidism often appears to be associated with congenital malformations. According to investigation data, $8,4 \%$ of 1420 children with congenital hypothyroidism had a prevalence of extrathyroidal congenital malformations, the majority were cardiac [4]. It is also observed that the incidence of congenital hypothyroidism is increased patients with Down syndrome. Screening programs for congenital hypothyroidism are now widely used in many well-developed countries. The method of screening involves collecting newborn blood samples on special filtered paper cards, that are then send to laboratories for testing. In case the disease is suspected, follow-up testing (e.g. measurement of thyroid hormone levels in blood serum) should be performed. Decreased level of T4 and increased level of TSH are in favor of congenital hypothyroidism. Diagnostic studies performed to determine underlying etiology include thyroid ultrasonography (manifestations of thyroid aplasia may be detected), radionuclide scans, measurements of serum thyroglobulin level as well as identification of antithyroid antibodies and genetic tests. Unfortunately, in countries with no screening programs available, diagnosis can be made only after clinical manifestations are developed. Prolonged jaundice in hypothyroidism can sometimes imitate clinical presentation of biliary atresia. Slow linear growth, large head with immature body proportion, radiological features of epiphyseal dysgenesis can suggest pituitary dwarfism. Final confirmation of the diagnosis can be made on the basis of clinical symptoms and signs as well as thyroid function tests.

There is a direct connection between the effectiveness of treatment and time when it was prescribed. Early replacement therapy in optimal doses assures quick settling of thyroid hormone levels and maintenance of clinical and biochemical euthyriodism. Treatment of choice is Levothyroxine, with its dosage depending on the severity of patient condition [5]. Normalization of T4 level is observed in up two weeks, TSH - in up to a month after therapy initiation. Measurements of thyroid hormone level should be performed frequently, namely, once a month during the first six month, then once in three months up till up to three years. Patient prognosis depends on different factors: therapy timing, initial dosage of Levothyroxine, severity of clinical manifestation. Based on studies, if onset of replacement therapy was between birth and 3 months, the mean IQ (Intelligence quotient) was 89, if treatment 
was started between 3 and 6 months of age, the mean IQ was 71, while if treatment did not start until after 6 months of age, the mean IQ dropped to 54 [6].

\section{Conclusion}

Prevention of permanent nervous system disorder and child healthy development can only be possible with early diagnostics and complex optimal treatment of congenital hypothyroidism.

\section{References}

1. Delange F (1997) Neonatal screening for congenital hypothyroidism: Results and perspectives. Horm Res 48(2): 51-61.

2. Grant DB, Smith I, Fuggle PW, Tokar S, Chapple J (1992) Congenital hypothyroidism detected by neonatal screening: Relationship between biochemical severity and early clinical features. Arch Dis Child 67(1): 87-90.
3. Hinton CF, Harris KB, Borgfeld l, Drummond M, Eaton R, et al. (2010) Trends in incidence rates of congenital hypothyroidism related to select demographic factors: Data from the United States, California, Massachusetts, New York and Texas. Pediatrics 125(Suppl 2): S37-47.

4. Olivieri A, Stazi MA, Mastroiacovo P, Fazzini C, Medda E, et al. (2002) A population-based study on the frequency of additional congenital malformations in infants with congenital hypothyroidism: Data from the Italian registry for congenital hypothyroidism (1991-1998). J Clin Endocrinol Metab 87(2): 557-562.

5. Selva KA, Mandel SH, Rien L, Sesser D, Miyahira R, et al. (2002) Initial treatment dose of L-thyroxine in congenital hypothyroidism. J Pediatr 141(6): 786-792.

6. Klein AH, Meltzer S, Kenny FM (1972) Improved prognosis in congenital hypothyroidism treated before age three months. J Pediatr 81(5): 912915.

For possible submissions Click below: 